\title{
Coxiella burnetii dormancy in a fatal ten- year multisystem dysfunctional illness: case report
}

Olga A. Sukocheva ${ }^{1,6}$, Jim Manavis², Tuck-Weng Kok ${ }^{3^{*}}$, Mark Turra ${ }^{4}$, Angelo Izzo ${ }^{5}$, Peter Blumbergs ${ }^{2}$ and Barrie P. Marmion ${ }^{1,4^{\wedge}}$

\begin{abstract}
Background: In a previous study of a $Q$ fever outbreak in Birmingham, our group identified a non-infective complex of Coxiella burnetii (C.b.) antigens able to survive in the host and provoked aberrant humoral and cellmediated immunity responses. The study led to recognition of a possible pathogenic link between C.b. infection and subsequent long-term post Q fever fatigue syndrome (QFS). This report presents an unusually severe case of C.b. antigen and DNA detection in post-mortem specimens from a patient with QFS.
\end{abstract}

Case presentation: We report a 19-year old female patient who became ill with an acute unexplained febrile encephalitis-like illness, followed by increasingly severe multisystem dysfunction and death 10 years later. During life, extensive clinical and laboratory investigations from different disciplinary stand points failed to deliver a definitive identification of a cause. Given the history of susceptibility to infection from birth, acute fever and the diagnosis of "post viral syndrome", tests for infective agents were done starting with C.b. and Legionella pneumophila. The patient had previously visited farms a number of times. Comprehensive neuropathological assessment at the time of autopsy had not revealed gross or microscopic abnormalities.

The aim was to extend detailed studies with the post-mortem samples and identify possible factors driving severe disturbance of homeostasis and organ dysfunction exhibited by the course of the patient's ten-year illness. Immunohistochemistry for C.b. antigen and PCR for DNA were tested on paraffin embedded blocks of autopsy tissues from brain, spleen, liver, lymph nodes (LN), bone marrow (BM), heart and lung. Standard H\&E staining of brain sections was unrevealing. Immuno-staining analysis for astrocyte cytoskeleton proteins using glial fibrillary acidic protein (GFAP) antibodies showed a reactive morphology. Coxiella antigens were demonstrated in GFAP immuno-positive grey and white matter astrocytes, spleen, liver, heart, BM and LN. PCR analysis (COM1/IS1111 genes) confirmed the presence of C.b. DNA in heart, lung, spleen, liver \& LN, but not in brain or BM.

Conclusion: The study revealed the persistence of C. b. cell components in various organs, including astrocytes of the brain, in a post-infection QFS. The possible mechanisms and molecular adaptations for this alternative C.b. life style are discussed.

Keywords: Q Fever, Antigen persistence, Chronic fatigue syndrome

\footnotetext{
* Correspondence: tuckweng.kok@adelaide.edu.au

'Deceased

${ }^{3}$ School of Biological Sciences, University of Adelaide, Adelaide, South

Australia

Full list of author information is available at the end of the article
} 


\section{Background}

Post infection fatigue syndrome has been described in patients exposed to facultative intracellular bacteria, including Coxiella burnetii (C.b.) - the causative agent of $\mathrm{Q}$ fever, as well as viruses [1]. The highly infectious (ca. one organism) rickettsia-like intracellular bacterium infects and multiplies in macrophages. In a previous report of a Q fever outbreak in Birmingham, our group identified a non-infective complex of C.b. antigens able to survive in the host and provoked aberrant humoral and cell-mediated immunity responses [2, 3]. The study led to recognition of a possible pathogenic link between infection and subsequent long-term post $\mathrm{Q}$ fever chronic fatigue syndrome (QFS).

We report a 19-year old female patient (coded initials BI) who became ill with an acute unexplained febrile encephalitis-like illness, followed by increasingly severe multisystem dysfunction and death ten years later (in 1996). During life, extensive clinical and laboratory investigations from different disciplinary stand points failed to deliver a definitive identification of a cause, but descriptive diagnoses, such as post infection fatigue syndrome, or just before death, Behçet's syndrome, were proposed. During her last 10 years, BI presented with severe fluctuating headaches, frequent dizziness, fever $40 \mathrm{C}+$, recurrent episodes of extensive pharyngeal ulcerations, muscular pain, persistent fatigue, joint pains, myoclonic seizures, quadriparesis, symptoms suggestive of meningism (neck rigidity and photophobia), bulbar paralysis and a range of gastrointestinal tract symptoms including abdominal pains, nausea, diarrhoea, bloating, oesophageal spasms as well as weight loss. It was noted that before the "encephalitis" there had been a history of inadequate, slow immune resolution on contracting various childhood infections.

At autopsy, standard histo-pathological methods revealed few abnormalities gross, or microscopic - an ulcer of the hard palate and very sparse patchy chronic inflammatory cell infiltration close to the atrio-ventricular node conducting system of the heart. Levels of inflammatory markers such as C-reactive protein (CRP) were within normal range, but with increased erythrocyte sedimentation rate. The negative organ and tissue profile was in sharp contrast to the severity of the symptomatic effects during life that included abnormal disabling fatigability, transient loss of consciousness ("blackouts"), loss of control over electrolyte balance and unexplained tissue oedema.

In view of BI's early history of abnormal susceptibility to infections, her acute fever and 'encephalitic' symptoms with a diagnostic label of severe "post viral infection fatigue syndrome", we suggested that her chronic persistent and severe multisystem disability might be an incidental systemic side effect ("bystander damage") of the specialised effector mechanisms, immune mediators and other gene products of facultative intracellular bacteria.

A major challenge remained that despite intense and dedicated investigative efforts from various medical subdisciplines it had not been possible to identify definitively the factors driving the severe disturbance of homeostasis and organ dysfunction exhibited by the course of BI's 10-year illness. In anticipation that a changed paradigm for post infection and related fatigue states would eventually emerge, BI's family had retained the paraffin wax-embedded or fixed slices of blocks of autopsy tissues from the patient's brain, spleen, liver, lymph nodes (LN), bone marrow (BM), heart, lung and other organs. The post mortem samples were submitted by the family for examination by extended techniques to search for possible changes in the brain using immunecytochemical markers for astrocytes and microglia. In the course of examining the paraffin wax-embedded tissues for possible neuropathology, prior Q fever infection was considered. BI had previously visited farms a number of times during childhood (see review [4]). Q fever antigens or specific antibodies had not been previously tested. This led to extended tests with staining for $Q$ fever antigens in the paraffin wax-embedded tissues. This report presents the immunohistochemical and PCR findings of C.b. antigens and DNA respectively in several organs, including astrocytes, from the patient's post mortem samples and discusses the association between persistence of the antigens, albeit noninfective, and QFS.

\section{Case presentation}

\section{C.b. antigen detection (Immunocytochemistry)}

Post-mortem samples were collected, formalin-fixed $(4 \%, v / v)$, and paraffin-embedded. Sections $(5 \mu \mathrm{m})$ of paraffin-embedded tissues were cut and mounted on APES coated (Superfrost plus) tissue slides and deparaffinised according to standard protocol. Control healthy (disease free) brain samples were collected from car accident trauma patient and used as an adequate standard control [5]. Slides were stained with routine Haematoxylin and Eosin $(\mathrm{H} \& \mathrm{E})$ for initial microscopic examination. For C.b. antigen detection, sections were pre-treated in citrate buffer (10 mM; pH 6.0) and heated by microwave and then allowed to cool to room temperature [5]. Antisera and negative sera were diluted in PBS. Immuno-staining was performed on selected slides with primary antibodies for the following markers: (1) C.b. Phase I and II antigens (rabbit polyclonal) or mouse Phase I monoclonal (a gift from CSL, Australia) [2, 3]; (2) polyclonal anti-GFAP (Santa Cruz Biotechnology); (3) monoclonal anti-lba-1 (Abcam). Negative controls consisted of sections immunostained as described 
below, but instead of overnight incubation with primary antibodies these were incubated with tris-buffered saline only (data not shown, but available). After washes in PBS/0.05 \% Tween-20 (Fisher Scientific), sections were incubated with 1:100 dilutions of anti-rabbit or antimouse secondary antibodies conjugated to fluorescein isothiocyanate (FITC) or phycoerythrin (PE) (Jackson lmmunoResearch Laboratories Inc). Nuclei were stained with Hoechst 33342 (Invitrogen). Fluorescence images were acquired and processed using the analysis software Olympus Soft Imaging System. For the images where comparisons of staining intensities were made, all images were acquired using the same attenuator and exposure settings.

\section{C.b. DNA test (PCR)}

DNA target nucleic acid sequences in tissue samples were tested by two separate PCR assays, one targeting the $27 \mathrm{kDa}$ outer membrane protein (COM1) and the other the insertion sequence (IS1111) [6]. These were tested with the DNA extracted from 2 paraffin-embedded tissue sections (10 mm thick) using Proteinase $\mathrm{K} 2 \mathrm{mg} / \mathrm{ml}$, SOS $0.5 \%$ w/v, and Qiagen (Hilden, Germany) DNA blood kit. Samples were amplified as described previously [6]. To minimize possible false positives, DNA extraction and PCR were performed carefully according to guidelines described previously $[7,8]$. A PCR result was considered negative (NEG, Table 1) when the $\mathrm{Ct}$ value was $\geq 37$. In a real time qPCR assay (quantitative PCR), the cycle threshold $(\mathrm{Ct})$ value is calculated to be the number of amplification cycles required for accumulated fluorescence signals in the reaction to exceed the background value. The higher the $\mathrm{Ct}$ value, the lower is the amount of amplified

Table 1 Quantitative PCR (qPCR) tests for C.b. DNA (IS1111a sequence) with patient Bl's paraffin-embedded tissues were tested in two PCR cyclers - RotorGene 1.7.73 (Corbett Research) and Roche LightCycler 4.05 (4.0.0.23)

\begin{tabular}{lll}
\hline Patient's post mortem specimens & Ct - RotorGene & Ct - LightCycler \\
\hline Heart & 23.34 & 25.65 \\
Lung & 36.37 & 36.46 \\
Brain & NEG & NEG \\
Bone marrow & NEG & NEG \\
Spleen/Liver & 25.15 & 27.08 \\
Lymph nodes & 22.47 & 24.80 \\
Control spleen & NEG & NEG \\
Control brain & NEG & NEG
\end{tabular}

The RotorGene Ct values shown are average of duplicate tests and LightCycler values from single tests. Non-template controls (NTC) were placed in between the samples and were negative $(\mathrm{Ct}>37)$. Thick sections of the post mortem tissues - for PCR tests - were cut with high level of precaution to minimize possible cross contamination at the Centre for Neurological Diseases (SA Pathology, South Australia) target nucleic acid sequence (Sequence Detection Systems v2.3 software - Applied Biosystems).

\section{Investigation of patient Bl's brain samples with immunohistochemical staining for astrocyte and distribution of C.b. antigen}

Given the history of susceptibility to infection from birth, the acute fever in 1986 and the diagnosis of "post (viral) infection syndrome", tests for infective agents were done starting with C.b. and Legionella pneumophila (L.p.). Patient BI had previously visited farms a number of times. Previous comprehensive neuropathological assessment at the time of autopsy in 1986 had not revealed gross or microscopic ( $\mathrm{H}$ \& $\mathrm{E}$ staining) abnormalities. The H\&E examination was repeated on fresh sections of brain from the patient and an unrelated control brain but no abnormalities were detected (data not shown). Immuno-peroxidase staining for glial fibrillary acidic protein (GFAP) showed prominent immune-staining of astrocytes and "beading" of the fibrillary processes compared with control brain sections which did not show such striking changes (Fig. 1a and b). Increased GFAP immuno-staining may be seen in many conditions that may be a non-specific reactive response of the astrocytes $[9,10]$.

Astrocytes in the occipital cortex and subcortical white matter from patient $\mathrm{BI}$ and control brain tissue were stained using GFAP antiserum with green-fluorescent (FITC) secondary antibodies, co-stained with Texas-red labelled monoclonal C.b. Phase 1 antiserum [2, 3] and viewed with confocal microscopy (Fig. 2). Astrocytes in the white and grey matter from patient BI (Fig. 2a and c, white and grey matter respectively) and control brain tissue (Fig. 2b and d) stained with anti-GFAP (green). Specific C.b. stained red and showed co-localization (yellow) with GFAP (green) protein (Fig. 2a). Staining with C.b. antisera revealed C.b. antigen complex in patient's astrocytes, supporting the possible involvement of $\mathrm{Q}$ fever infection in the illness. Note that only a minority of astrocytes contained the C.b. Phase 1 antigen complex. C.b. antigens were also detected in cells from hippocampal white matter using dual staining with anti-GFAP and confocal microscopy (data not shown). White and grey matter staining for C.b. was negative in a control (uninfected) brain. Microglia, the innate immune cells mediating inflammatory responses in the CNS [10-12] were identified using the specific Iba-1 marker [13, 14]. Double immune-staining of the Iba-1 reactive cells with C.b. antisera was negative (data not shown).

\section{Distribution of C.b. antigens in other tissue samples from patient BI}

Tissue samples from several lymphoid organs and those previously determined as targets for C.b. were further 


\section{A Occipital cortex}
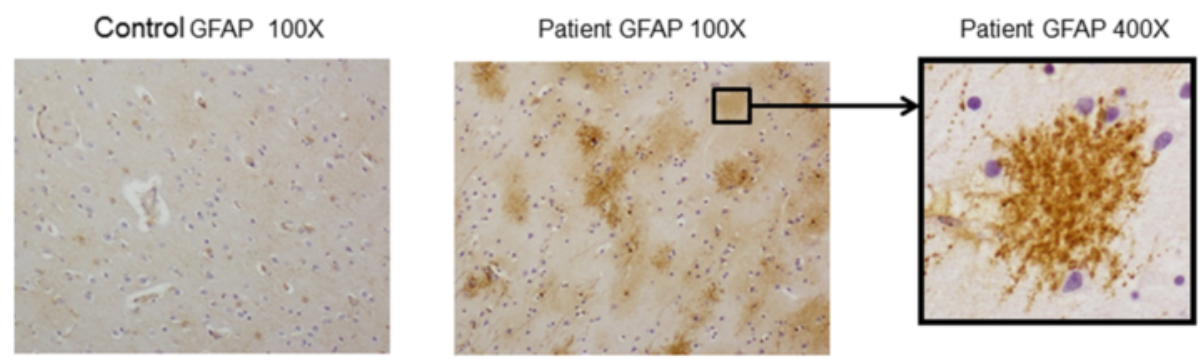

B Occipital subcortical white matter
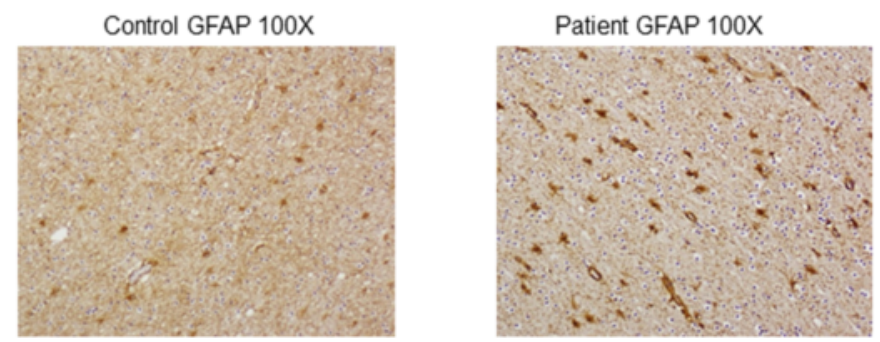

Fig. 1 Assessment of Patient BI and control astrocyte GFAP immune-staining in occipital cortex and occipital subcortical white matter using peroxidase staining technique and anti-GFAP antibodies as described in Methods. a Peroxidase staining with GFAP in samples from BI occipital cortex showing a reactive astrocyte with numerous "beaded" processes (400X). b Peroxidase staining with GFAP in BI samples from occipital subcortical white matter showing increased GFAP immune-staining and a reactive phenotype. Representative photographs are shown

stained using specific antisera. Figures 3 and 4 show C.b. Phase 1 LPS antigens detected in the macrophages of the patient's bone marrow (Fig. 3a), liver (Fig. 3b), lymph node (Fig. 3c), spleen (Fig. 3d) and heart (Fig. 4). Strong staining with the monoclonal antibody to C.b. Phase 1 LPS antigen was detected in both immuno-peroxidase (see Fig. 4) and IFA reactions (data not shown). However, the pattern was markedly different to that commonly described in Q fever endocarditis (early or late stage) where the valve lesions show fibrosis and calcification in the late stage [15]. The paucity of inflammatory cells including monocyte/macrophage lineage and the rapid decline of infective coxiella leaving antigen LPS complex has frequently been remarked, as are low levels of genomic DNA [16].

\section{C.b. DNA results}

PCR tests for C.b. genomic DNA sequences (COM1 and IS1111a genes [6]) in separate tests did not detect the presence of specific DNA in patient BI's brain, but nevertheless revealed substantial C.b. DNA in heart, lung, spleen and liver (Table 1) - in agreement with the results found during IFA and peroxidase staining with C.b. Phase 1 antisera (Figs. 3 and 4). PCR tests for COM1 and IS1111a genes showed similar results but only $\mathrm{Ct}$ values for the latter gene are shown in Table 1 . The negative PCR result in the brain tissues may be due to DNA levels below the limit of detection (10-20 copies/ml, unpublished data).

\section{Conclusions}

The scope and overall interpretation of our report on the re-evaluation of the fixed, paraffin-embedded tissue samples taken at patient BI's autopsy are necessarily limited by the absence of diagnostic results of any serological or other tests for candidate infective organisms at the time of the "viral encephalitis" in 1986 (see review [17]). Nevertheless, taken together, the extended laboratory tests with C.b. specific monoclonal antibodies and PCR (COM1 and IS1111a genes) on a range of post mortem specimens suggest that the most compelling and coherent explanation of BI's illness from 1986 to 1996, is one of a severe attack of primary Q fever and a subsequent multisystem organ dysfunction with dissemination of the coxiella throughout the body, ending in 1996 with cardiac and cerebral dysfunction i.e., a complex, severe idiopathic illness labelled descriptively at the time as "post (viral) infection fatigue syndrome" (PIFS).

An epidemiological and clinical association between laboratory-confirmed primary acute $\mathrm{Q}$ fever infection and a post infection fatigue syndrome was described in 1996/8 in meat workers and farmers in Australia and in patients in the wind-borne sheep-associated Q fever outbreak in the non-occupationally exposed populations of 


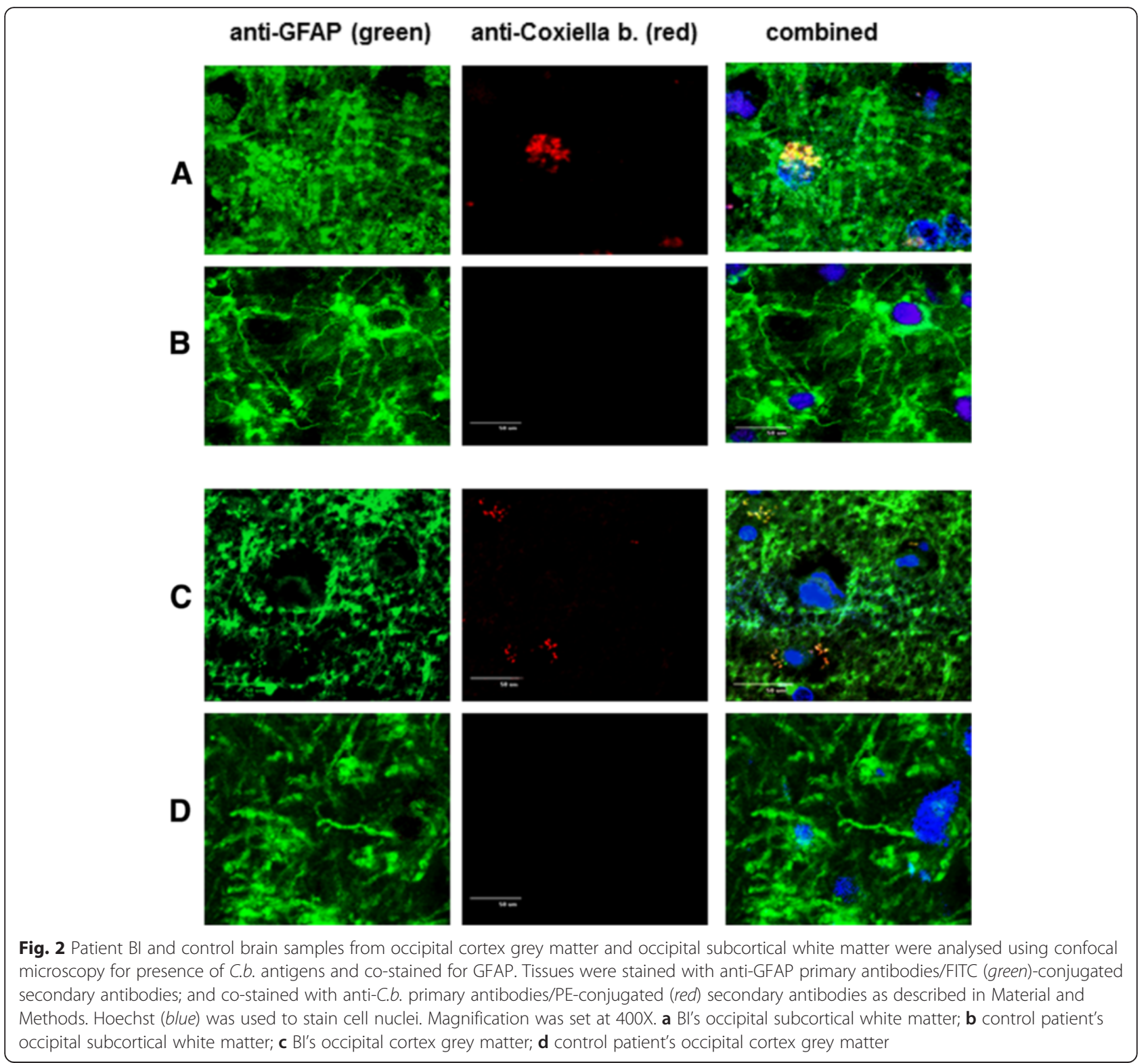

South Birmingham UK $[18,19]$. At first the post Q fever syndrome (QFS) tended to be interpreted as a psychogenic response by patients to a chronic debilitating illness. Since then, however, the association has been described in many countries. For example, an independent follow up of patients in a goat-associated Q fever outbreak in Alberta, Canada included a patient with severe unresolved cerebral symptoms but C.b. was not isolated from the CNS [20]. The recent major prevalence ( $>4000$ cases) of goat-associated Q fever in the Netherlands, allowed well-controlled surveys for "post infection fatigue syndromes" and again confirmed prevalence of QFS and vascular complications after an initial infection [21].
Some of the scepticism and difficulty of acceptance of QFS may have rested on a mistaken assumption that the model of infection with the small intracellular bacterium, Coxiella burnetii, would follow a familiar set of characteristics of invasion, bacteraemia, inflammatory responses, finally resolution with rising antibody levels, acquired cellular immune responses and elimination of the invading organism [2]. Investigations of QFS by the Q fever Research Group in Adelaide (Australia) have not supported this simple model [2,3]. Notably, long term persistence in the host of various components of the coxiella antigens shown e.g. LPS Phase I antigen, coxiella genomic DNA sequences and low level lgG class antibody in the human host to C.b. Phase I \& II antigens 


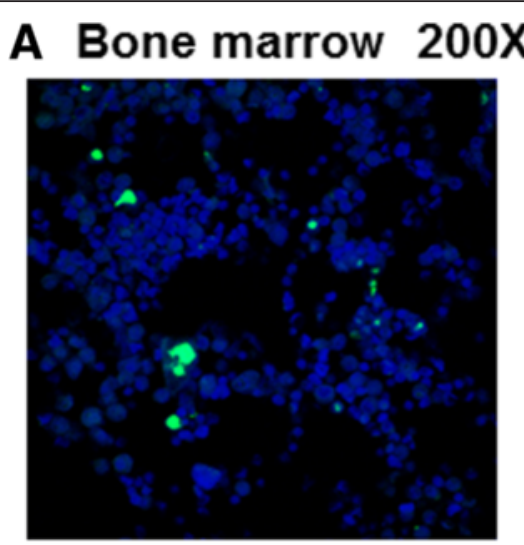

\section{B Liver 400X}

\section{LNs 400X}

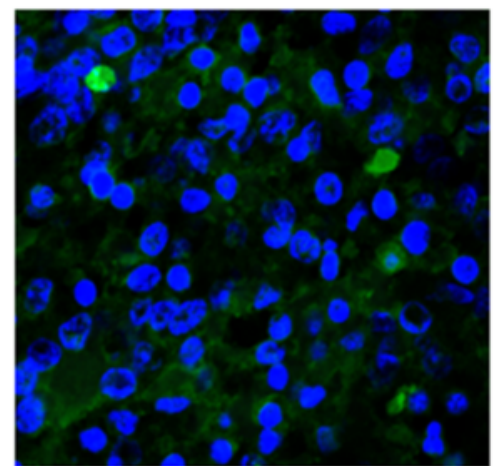

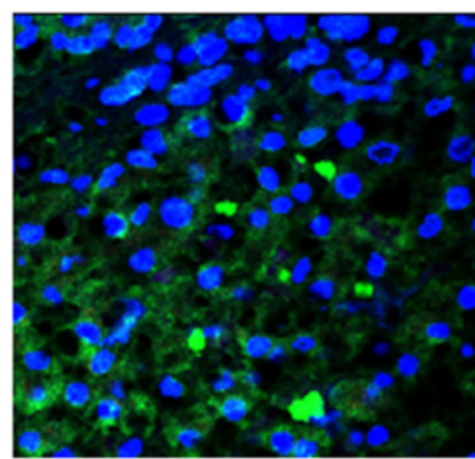

D Spleen $600 x$

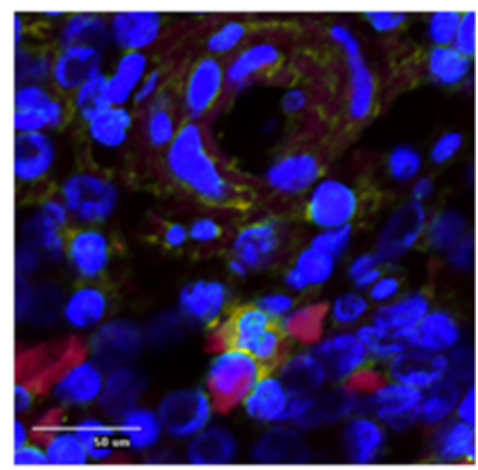

Alexa-red-CD11c

FITC-green- coxiella Hoechst-Blue-nucleus

Fig. 3 Presence of C.b. complexes in patient Bl's lymphoid tissues were assessed using confocal immunostaining assay: a Bone marrow, b Liver, c LNs, d Spleen. Alexa-red was used to identify CD11c (dendritic cells - only for D). C.b. specific antibody and FITC (green) secondary antibody were used to detect presence of C.b. and Hoechst (blue) stain defines nuclei

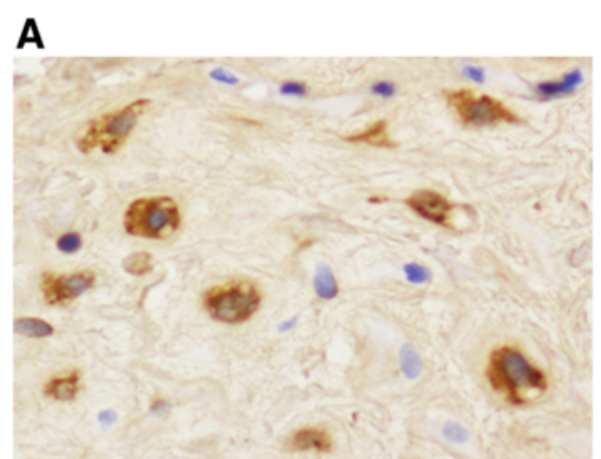

B

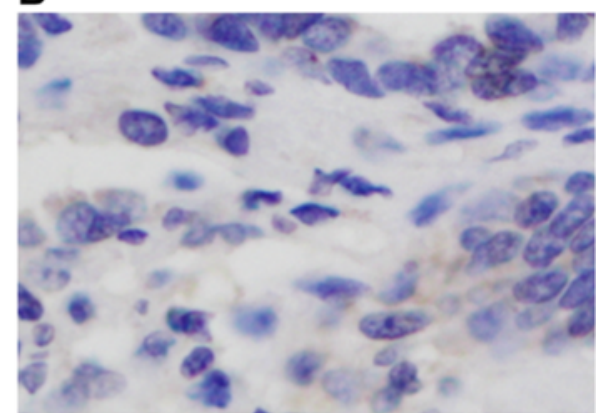

Fig. 4 a Immuno-peroxidase staining of myocardium - close to mitral valve - with specific monoclonal antibodies for presence of C.b. antigens X400. b Negative control 
were often present in QFS patients more than 10 years after the original infection [3]. On the other hand, in perplexing contrast, efforts to demonstrate consistent presence of the readily serial cultivable coccobacilliary form of the coxiella (small cell variant, SCV) in such materials have mostly failed [2, 3, 18-20].

The SCV, the infectious particle, is found in the environment and is metabolically inactive. When internalized into a cell the SCV is transformed into the metabolically active large cell variant (LCV) [22]. The LCV develops in response to acidification of the endosome and is associated with the development of the parasitophorous vacuole (PV) by the host cell that allows the organism to replicate. There is evidence to suggest that the highly acidic PV is regulated by the LCV and provides an excellent environment for it to grow. Further studies will be required to determine the relationship of the SCV with the PV [23, 24].

In the acute phase of $\mathrm{Q}$ fever, C.b. SCV is widely distributed in the host but in general they do not persist as they transition to the LCV form. In contrast noninfective C.b. components - complexes of Phase 1 LPS, antigens, DNA sequences or insertion elements have been detected in PBMC, bone marrow aspirates or valve vegetations $[2,3,18,19]$. Such residual complexes of coxiella cell components persisted for 12-17 years after infection in the patients in the Birmingham UK Q fever outbreak [3]. Despite some partial "immune" responses the C.b. materials are not cleared and IL-2 is downregulated, IFN- $\gamma$ responses distorted and IL- 6 responses are enhanced $[25,26]$. The effect is supported by robust ability of C.b. to interfere with pathogen-initiated apoptosis upon infection of mammalian cells $[27,28]$ and by the activation of type IV intracellular organism effector repertoire $[29,30]$.

In summary, the recorded history, clinical findings and indices for patient BI, and the series of reports on QFS patients from the Adelaide $\mathrm{Q}$ fever Research Group in Australia and Birmingham UK, support a hypothesis that there is an association to be tested between persistence of C.b. components - as described above - the modified macrophage as a PV. The terms PIFS or QFS - while correctly labelling a major clinical presentation - do not do justice to the range of different effects or multiple host organ systems involved, particularly as illustrated in an unusual and exceptionally severe case such as patient BI.

\section{Ethics approval and consent}

Not applicable.

\section{Consent for publication}

Written informed consent was obtained from the patient's parent for publication of this Case Report. A copy of the written consent is available for review by the Editor of this journal.

\section{Availability of data and materials}

All data supporting the findings are within the manuscript.

\section{Abbreviations \\ BM: bone marrow; C.b.: Coxiella burnetii; FITC: fluorescein isothiocyanate: \\ GFAP: glial fibrillary acidic protein; IFA: immunofluorescent antibody; \\ IFN: interferon; LCV: large cell variant; LN: lymph nodes; \\ LPS: lipopolysaccharide; PCR: polymerase chain reaction; PIFS: post (viral) infection fatigue syndrome; PV: parasitophorous vacuole; QFS: Q fever fatigue syndrome; SCV: small cell variant.}

\section{Competing interests}

The authors declare that they have no competing interests.

\section{Authors' contributions}

OS, T-WK, PB and BPM designed, analysed and interpreted the data; OS, JM, TWK, MT and PB did the immune-histochemical, IF and PCR tests and analysis; OS, TWK, Al, PB and BPM drafted the manuscript. All authors approved the final manuscript.

\section{Acknowledgements}

Professor Barrie P. Marmion passed away in July 2014. The authors would like to express our deep respect for the enormous contributions that Professor BPM made to $Q$ Fever research, to the introduction of the $Q$ Fever vaccine in Australia and the establishment of major virology research - in particular Hepatitis B. Two of his postgraduate students (Al, T-WK) and postdoctoral scientist (OS) are profoundly honoured to have been mentored by BPM.

\section{Funding}

The authors are grateful to Mrs C. Hunter and Alison Hunter Memorial Foundation in Partnership with the National Centre for Neuroimmunology and Emerging Diseases (NCNED), Griffith University, Queensland, Australia for generous financial support of this investigation.

\section{Author details}

'Q Fever Research Group (1993-2009), Hanson Institute, Adelaide, South Australia. ${ }^{2}$ Centre for Neurological Diseases, SA Pathology, Adelaide, South Australia. ${ }^{3}$ School of Biological Sciences, University of Adelaide, Adelaide, South Australia. ${ }^{4}$ Microbiology and Infectious Diseases Laboratory, SA Pathology, Adelaide, South Australia. ${ }^{5}$ Colorado State University, Colorado, USA. ${ }^{6}$ School of Health Sciences, Flinders University, Bedford Park, South Australia.

Received: 19 October 2015 Accepted: 6 April 2016

Published online: 18 April 2016

\section{References}

1. Bansal AS, Bradley AS, Bishop KN, Kiani-Alikhan S, Ford B. Chronic fatique syndrome, the immune system and viral infection. Brain Behav Immun. 2012:26:24-31.

2. Marmion BP, Sukocheva O, Storm PA, Lockhart M, Turra M, et al. Q fever: persistence of antigenic non-viable cell residues of Coxiella burnetii in the host-implications for post Q fever infection fatigue syndrome and other chronic sequelae. QJM. 2009;102:673-84.

3. Sukocheva OA, Marmion BP, Storm PA, Lockhart M, Turra M, et al. Longterm persistence after acute $\mathrm{Q}$ fever of non-infective Coxiella burnetii cell components, including antigens. QJM. 2010;103:847-63.

4. Georgiev M, Afonso A, Neubauer $H$, Needham $H$, Thiery $R$, et al. Q fever in humans and farm animals in four European countries, 1982 to 2010. Euro Surveill 2013;18(8).

5. Finnie JW, Blumbergs PC, Manavis J, Vink R. Pattern of cerebrospinal immediate early gene $c$-fos expression in an ovine model of non-accidental head injury. J Clin Neurosci. 2013;20:1759-61.

6. Turra M, Chang G, Whybrow D, Higgins G, Qiao M. Diagnosis of acute Q fever by PCR on sera during a recent outbreak in rural south Australia. Ann N Y Acad Sci. 2006:1078:566-9.

7. Marmion BP, Storm PA, Ayres JG, Semendric L, Mathews L, et al. Long-term persistence of Coxiella burnetii after acute primary Q fever. QJM. 2005;98:7-20.

8. Yuasa Y, Yoshiie K, Takasaki T, Yoshida H, Oda H. Retrospective survey of chronic Q fever in Japan by using PCR to detect Coxiella burnetii DNA in paraffin-embedded clinical samples. J Clin Microbiol. 1996;34:824-7. 
9. Huber AK, Duncker PC, Irani DN. Immune responses to non-tumor antigens in the central nervous system. Front Oncol. 2014;4:328.

10. Lehnardt S. Innate immunity and neuroinflammation in the CNS: the role of microglia in Toll-like receptor-mediated neuronal injury. Glia. 2010;58:253-63.

11. Hanisch UK, Kettenmann H. Microglia: active sensor and versatile effector cells in the normal and pathologic brain. Nat Neurosci. 2007;10:1387-94.

12. Ransohoff RM, Brown MA. Innate immunity in the central nervous system. J Clin Invest. 2012;122:1164-71.

13. Ito D, Imai Y, Ohsawa K, Nakajima K, Fukuuchi Y, et al. Microglia-specific localisation of a novel calcium binding protein, Iba1. Brain Res Mol Brain Res. 1998:57:1-9.

14. Torres-Platas SG, Comeau S, Rachalski A, Bo GD, Cruceanu C, et al. Morphometric characterization of microglial phenotypes in human cerebral cortex. J Neuroinflammation. 2014;11:12.

15. Benoit M, Ghigo E, Capo C, Raoult D, Mege JL. The uptake of apoptotic cells drives Coxiella burnetii replication and macrophage polarization: a model for Q fever endocarditis. PLoS Pathog. 2008:4:e1000066.

16. Raoult D, Casalta JP, Richet H, Khan M, Bernit E, et al. Contribution of systematic serological testing in diagnosis of infective endocarditis. J Clin Microbiol. 2005;43:5238-42.

17. Morris G, Maes M. Case definitions and diagnostic criteria for Myalgic encephalomyelitis and chronic fatigue syndrome: from clinical-consensus to evidence-based case definitions. Neuro Endocrinol Lett. 2013;34:185-99.

18. Ayres JG, Wildman M, Groves J, Ment J, Smith EG, et al. Long-term follow-up of patients from the $1989 \mathrm{Q}$ fever outbreak: no evidence of excess cardiac disease in those with fatigue. QJM. 2002:95:539-46.

19. Marmion BP, Shannon M, Maddocks I, Storm P, Penttila I. Protracted debility and fatigue after acute Q fever. Lancet. 1996;347:977-8.

20. Hatchette TF, Hayes M, Merry H, Schlech WF, Marrie TJ. The effect of C burnetii infection on the quality of life of patients following an outbreak of Q fever. Epidemiol Infect. 2003;130:491-5.

21. Schneeberger PM, Wintenberger C, van der Hoek W, Stahl JP. Q fever in the Netherlands - 2007-2010: what we learned from the largest outbreak ever. Med Mal Infect. 2014;44:339-53

22. Voth DE, Heinzen RA. Lounging in a lysosome: the intracellular lifestyle of Coxiella burnetii. Cell Microbiol. 2007;9:829-40.

23. Larson CL, Beare PA, Voth DE, Howe D, Cockrell DC, et al. Coxiella burneti effector proteins that localize to the parasitophorous vacuole membrane promote intracellular replication. Infect Immun. 2015;83:661-70.

24. van Schaik EJ, Chen C, Mertens K, Weber MM, Samuel JE. Molecular pathogenesis of the obligate intracellular bacterium Coxiella burnetii. Nat Rev Microbiol. 2013;1 1:561-73.

25. Helbig K, Harris R, Ayres J, Dunckley H, Lloyd A, et al. Immune response genes in the post-Q-fever fatigue syndrome, $\mathrm{Q}$ fever endocarditis and uncomplicated acute primary Q fever. QJM. 2005;98:565-74.

26. Penttila IA, Harris RJ, Storm P, Haynes D, Worswick DA, et al. Cytokine dysregulation in the post-Q-fever fatigue syndrome. QJM. 1998;91:549-60.

27. Luhrmann A, Roy CR. Coxiella burnetii inhibits activation of host cell apoptosis through a mechanism that involves preventing cytochrome $c$ release from mitochondria. Infect Immun. 2007:75:5282-9.

28. Voth DE, Heinzen RA. Sustained activation of Akt and Erk1/2 is required for Coxiella burnetii antiapoptotic activity. Infect Immun. 2009;77:205-13.

29. Beare PA, Gilk SD, Larson CL, Hill J, Stead CM, et al. Dot/lcm type IVB secretion system requirements for Coxiella burnetii growth in human macrophages. MBio. 2011;2:e00175-00111.

30. Pan X, Luhrmann A, Satoh A, Laskowski-Arce MA, Roy CR. Ankyrin repeat proteins comprise a diverse family of bacterial type IV effectors. Science. 2008;320:1651-4.

\section{Submit your next manuscript to BioMed Central and we will help you at every step:}

- We accept pre-submission inquiries

- Our selector tool helps you to find the most relevant journal

- We provide round the clock customer support

- Convenient online submission

- Thorough peer review

- Inclusion in PubMed and all major indexing services

- Maximum visibility for your research

Submit your manuscript at www.biomedcentral.com/submit
Biomed Central 